Pleione 12(1): 118 - 127. 2018.

\title{
New Additions to the angiospermic flora of Nagaland, India
}

\author{
K. Ravikumar ${ }^{1}$, N. Dhatchanamoorthy, A. C. Tangavelou, \\ T. S. Suma and S. Noorunnisa Begum
}

\author{
National Herbarium of Medicinal Resources used in Indian Medical Systems, Institute of Trans- \\ Disciplinary Health Sciences and Technology (ITDHST), Foundation for Revitalisation of Local \\ Health Traditions (FRLHT), 74/2 Jarakabande Kaval, Attur Post, Via - Yelahanka, \\ Bangalore - 560 064, Karnataka, India \\ ${ }^{1}$ Author for correspondence: e-mail: ravikumarfrlht@gmail.com
}

[Received 02.04.2018; Revised \& accepted 26.06.2018; Published 30.06.2018]

\begin{abstract}
Botanical survey conducted to the North-Eastern states of India has resulted in the addition of 13 angiospermic taxa [Anaphalis royleana DC., Boehmeria penduliflora Wedd. ex D.G.Long, Byttneria pilosa Roxb., Cirsium verutum (D.Don) Spreng., Glochidion multiloculare var. pubescens Chakrab. \& Gangop., Inula cuspidata (Wall. ex DC.) C.B.Clarke, Myriactis wallichii Less., Phyllanthus rheedei Wight, Sarcochlamys pulcherrima Gaudich, Swertia ciliata (D. Don ex G. Don) B.L. Burtt, Symplocos sumuntia Buch.-Ham. ex D. Don, Tetrastigma serrulatum (Roxb.) Planch. and Uraria crinita (L.) DC.] which form new distributional record to the State of Nagaland in India. The information is presented here with brief botanical description, distribution, phenology, habitat and photographs.
\end{abstract}

Key words: Angiosperm, Flora, Nagaland, New additions, North-East India

\section{INTRODUCTION}

To strengthen the National Herbarium of Medicinal Plants (FRLH) in Foundation for Revitalisation of Local Health Traditions (FRLHT), Bengaluru, India, the botanical team had undertaken floristic surveys in the forests of Dimapur, Peren and Kohima districts of Nagaland state during September 2015. During this extensive field exploration, the authors have collected several plant specimens (200 voucher specimens), of which, based on critical study, 13 plant taxa are interesting and show new distributional report to Nagaland state. These specimens were confirmed by referring various literature (Hooker 1872 - 1897; Kanjilal et al. 1934 - 1940; Balakrishnan 1981, 1983; Chowdhery \& Wadhwa 1984; Haridasan \& Rao, 1985, 1987; Sanjappa 1992; Chaudhuri 1993; Sharma \& Sanjappa 1993; Hajra et al. 1995a,b; Chauhan 1996; Chowdhery et al. 1996, 2008; Polunin \& Stainton 1997; Singh et al. 2000a,b; Singh 2001; Kumar \& Singh 2001; Singh et al. 2002; Balakrishnan et al. 2012) and also the specimens were compared with authentic herbarium specimens deposited at Botanical Survey of India, Shillong, Kolkata and Kew Digital Herbarium. All the 13 plant taxa namely Anaphalis royleana, Boehmeria penduliflora, Byttneria pilosa, Cirsium verutum, Glochidion multiloculare var. pubescens, Inula cuspidata, Myriactis wallichii, Phyllanthus rheedei, Sarcochlamys pulcherrima, Swertia ciliata, Symplocos sumuntia, Tetrastigma serrulatum and Uraria crinita are presented here in alphabetical order with updated nomenclature, family, phenology, habitat, distribution and specimens examined. All the voucher specimens are deposited at FRLH Herbarium, Bengaluru. 


\section{TAXONOMIC ENUMERATION}

Anaphalis royleana DC., Prodr. 6: 272. 1838; Hook.f., Fl. Brit. India 3: 280. 1881; Hajra et al., Fl. India 13: 71 \& 72. 1995; Chowdhery et al., Fl. Arunachal Prad. 2: 4. 2008. (Asteraceae) [Plates 1a \& 2a]

Herbs, up to $50 \mathrm{~cm}$ high; branches erect, white tomentose. Leaves sessile, adnate to stem, linear-oblong or oblong-lanceolate, $2-5 \times 0.2-0.4 \mathrm{~mm}$, decurrent at base, entire, recurved at margins, acute at apex, 1-veined, upper side appressed, scaberulous hairy above, white tomentose beneath. Capitula c. $9 \mathrm{~mm}$, densely arranged in corymbs; peduncles greyish white tomentose, as long as inflorescence. Involucre c. $5 \mathrm{~mm}$ long; phyllaries white, glabrous; outer ones ovate, shorter than florets; middle ones ovate-oblong, longer than florets; inner ones linear-oblong, as long as disc. Achenes young, flat.

Flowering \& Fruiting: August - October.

Habitat: Less common, found along the edges of evergreen forest.

Distribution: Bhutan, China, northern Myanmar, Nepal, Pakistan, Tibet, Taiwan and India. In India, this species is reported from Arunachal Pradesh, Jammu \& Kashmir, western and eastern Himalaya, Sikkim and now collected from Nagaland.

Specimens examined: INDIA: Nagaland, Kohima district, Dzuleke village, $1810 \mathrm{~m}, 27^{\text {th }}$ September 2015, K. Ravikumar, T.S. Suma \& A.C. Tangavelou 119375 (FRLH).

Boehmeria penduliflora Wedd. ex D.G. Long, Notes Roy. Bot. Gard. Edinburgh. 40: 130. 1982; Chauhan, Contrib. Fl. Namdapha, Arunachal Prad. 291. 1996. B. penduliflora Wedd. in Ann. Sci. Nat. ser. 4, 1: 199. 1854, nom. nud. B. macrophylla D.Don, Prodr. Fl. Nep. 60. 1825, auct. non Hornem; Hook.f., Fl. Brit. India 5: 577. 1888; Kanjilal et al., Fl. Ass. 4: 288. 1940. (Urticaceae) [Plates 1b \& 2b]

Shrubs, up to $3 \mathrm{~m}$ high; branches 4 -angled to terete, densely strigose. Leaves narrowly lanceolate, $c$. $25 \times 4.5 \mathrm{~cm}$, rounded at base, serrulate at margin, caudate-acuminate at apex, chartaceous, 3-nerved at base and reaching up to apex, upper side sparsely pubescent along nerves, brownish, lower side strongly rugose and sparsely strigose. Female spikes unbranched, up to $40 \mathrm{~cm}$ long.

Flowering \& Fruiting: August - November

Habitat: Dry deciduous forests near small streamlets.

Distribution: Bhutan, China, Myanmar, Nepal, Thailand, Vietnam and India. In India, it is reported from Arunachal Pradesh, Meghalaya, Sikkim and now from Nagaland state.

Specimen examined: INDIA:Nagaland, Dimapur district, Chumukedima hills, $215 \mathrm{~m}, 26^{\text {th }}$ September 2015, K. Ravikumar, T.S. Suma \& A.C. Tangavelou 119332 (FRLH).

Byttneria pilosa Roxb., Fl. India 1: 618, 381. 1824; Hook.f., Fl. Brit. India 1: 377. 1874; Kanjilal et al., Fl. Ass. 1: 160. 1934; Sharma et al., Fl. India 3: 414. 1993; Singh et al., F1. Manipur 1: 176. 2000. (Sterculiaceae) [Plates 1c \& 2c]

Climbing shrubs; branches grooved, brownish when dry, stellate hairy. Leaves orbicular or ovate, shortly 3-5 lobed, $12-20 \times 11-19 \mathrm{~cm}$, cordate at base, serrulate at margin, acuminate at apex, membranous, stellate-pilose on both sides, c. 9-nerved at base; petiole grooved or striated, c. $15 \mathrm{~cm}$ long, tomentose. Inflorescence a cyme, axillary, few-flowered. Flowers small, many branched, pubescent; bracteoles subulate-slender, $c .5 \mathrm{~mm}$ long. Sepals subulateovate, $c .3 \times 1 \mathrm{~mm}$ long, glabrous above, hairy below. Petals yellow, incurved, shorter than 

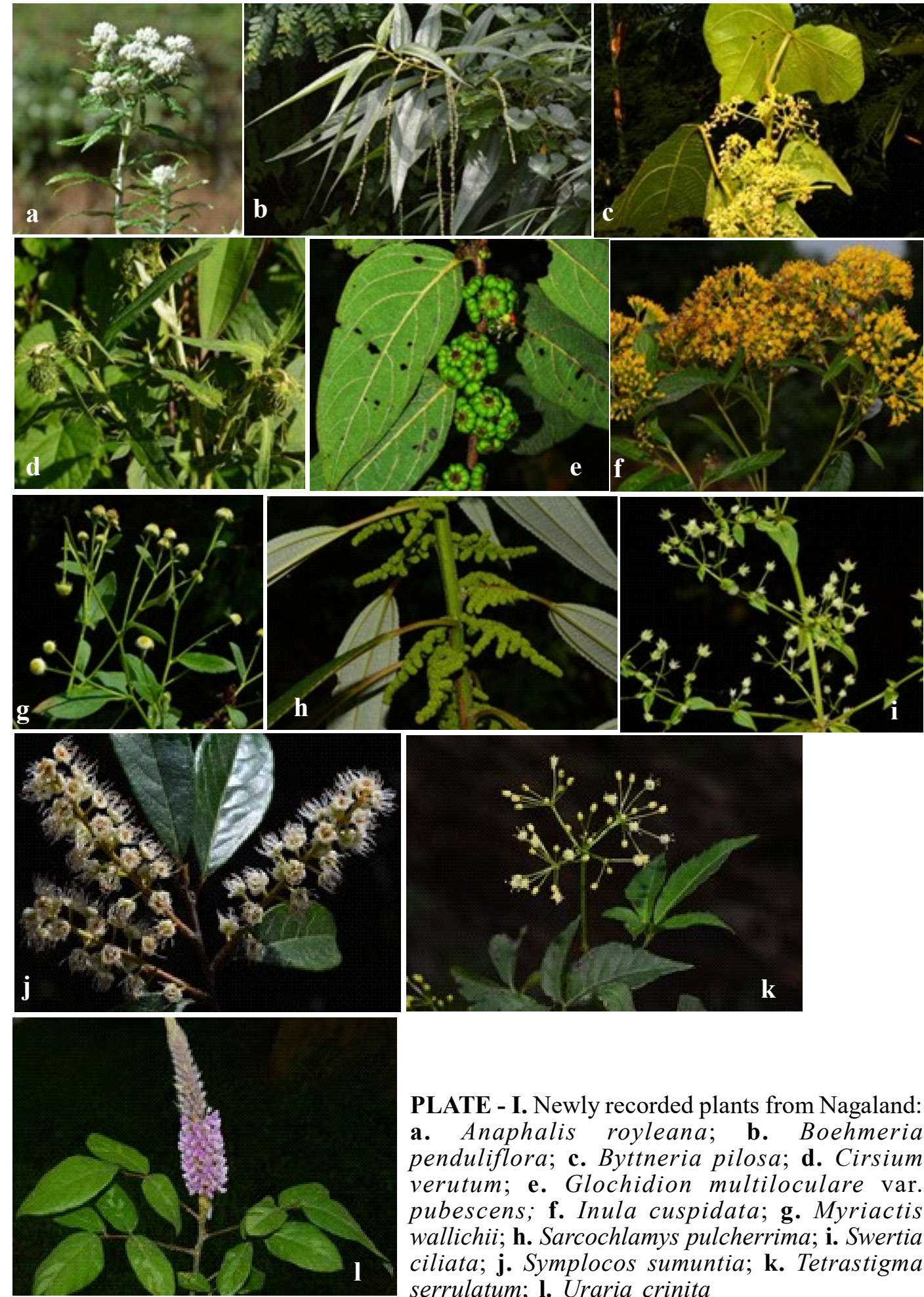

PLATE - I. Newly recorded plants from Nagaland: a. Anaphalis royleana; b. Boehmeria penduliflora; c. Byttneria pilosa; d. Cirsium verutum; e. Glochidion multiloculare var. pubescens; f. Inula cuspidata; g. Myriactis wallichii; h. Sarcochlamys pulcherrima; i. Swertia ciliata; j. Symplocos sumuntia; k. Tetrastigma serrulatum; l. Uraria crinita 
sepals, lobes obtuse. Staminodes partly connate, $c .1 \mathrm{~cm}$ long. Ovary globose, $c .1 \mathrm{~mm}$ long, verrucose; style about as long as the ovary.

Flowering: August - September

Habitat: Deciduous forest, commonly found along the periphery of the forest.

Distribution: Bangladesh, Indonesia, Laos, Malaysia, Myanmar, Thailand, Vietnam and India. In India, it is reported from Assam, Meghalaya, Manipur, Sikkim, West Bengal and now reported from Nagaland.

Specimen examined: INDIA: Nagaland, Kohima district, Mezoma, $360 \mathrm{~m}, 29^{\text {th }}$ September 2015, K. Ravikumar, T.S. Suma \& A.C. Tangavelou 119438 (FRLH).

Cirsium verutum (D. Don) Spreng., Syst. Veg., ed. 16.3: 370. 1826; Hajra et al., Fl. India 12: 171. 1995. Cnicus verutus D. Don, Prodr. Fl. Nepal. 167. 1825. C. involucratum DC., Prodr. 6: 639. 1838. C. involucratum var. horrida (Hook.f.) Balakr., Fl. Jowai 1: 256. 1981. C. involucratus var. horrida, Hook.f., Fl. Brit. India 3: 326. 1881. (Asteraceae) [Plates 1d \& 2d]

Shrubs, up to $3 \mathrm{~m}$ tall; branches terete, shallowly winged, hairy. Leaves sessile, oblanceolate, c. $15 \times 9 \mathrm{~cm}$, pinnati-partite, semi-amplexicaule at base; lateral spinules 3-5 mm long; apical spine $c .5 \mathrm{~mm}$ long, basal teeth sometimes spiniform. Bracts ovate, $c .10 \times 5 \mathrm{~mm}$, spiny at margin. Capitula corymbose. Involucre campanulate, $3-5 \times c .5 \mathrm{~mm}$, glabrous or sparsely silky. Phyllaries imbricate in 5-7 rows; outer and middle phyllaries $1.5-2.5 \mathrm{~mm}$, florets bisexual. Corolla whitish, c. $2.8 \mathrm{~cm}$ long. Achenes subflattened, dark, c. $3.5 \mathrm{~mm}$ long. Pappus bristles brownish or pale whitish, up to $2.5 \mathrm{~cm}$ long.

Flowering \& Fruiting: July - September

Habitat: Common in evergreen forests.

Distribution: Afghanistan, Bhutan, China, Nepal, Pakistan and India. In India, it is reported from Jammu \& Kashmir, Himachal Pradesh, Uttar Pradesh, Sikkim, Meghalaya and now collected from Nagaland.

Specimen examined: INDIA: Nagaland, Peren district, Benreu village (near Government Middle School), $1890 \mathrm{~m}, 29^{\text {th }}$ September 2015, K. Ravikumar, T.S. Suma \& A.C. Tangavelou 119487 (FRLH).

Glochidion multiloculare (Rottler ex Willd.) Voigt var. pubescens Chakrab. \& Gangop. in J. Econ. Taxon. Bot. 14: 720. 1990; Balakrishnan et al. Fl. India 23: 427. 2012. (Phyllanthaceae) [Plates 1e \& 2e]

Trees, up to $8 \mathrm{~m}$ tall; stem hallow inside. Leaves elliptic-lanceolate, $8-11 \times 3-4.5 \mathrm{~cm}$, cuneate at base, entire at margins, acute-acuminate at apex; lateral nerves $c .7$ pairs, appressed hairy above, pubescent below; petioles up to $6 \mathrm{~mm}$ long. Fruiting inflorescences fascicled in leaf axil; peduncles subsessile, c. $3 \mathrm{~mm}$. Capsules depressed globose, 8-10 grooved, $c .1 \times 1 \mathrm{~cm}$, glabrous; seeds subglobose, $c .5 \times 2 \mathrm{~mm}$, subacute at apex, glabrous, red when mature.

Fruiting: July - September.

Habitat: Occasional in evergreen forest.

Distribution: Endemic to tropical forests of India and reported from Assam, Sikkim and now recorded from Nagaland. 
Specimen examined: INDIA: Nagaland, Kohima district, Dzuleke village, $1900 \mathrm{~m}, 27^{\text {th }}$ September 2015, K. Ravikumar, T.S. Suma \& A.C. Tangavelou 119420 (FRLH).

Inula cuspidata (Wall. ex DC.) C.B. Clarke, Compos. Ind. 125. 1876; Hook.f., Fl. Brit. India 3: 296, 1881; Hajra et al., Fl. India 13: 15. 1995. Amphiraphis cuspidata DC., Prodr. 5: 343. 1836. (Asteraceae) [Plates 1f \& 2f]

Herbs, up to $80 \mathrm{~cm}$ high; branches striate; young stems minutely pubescent. Leaves ellipticlanceolate, $6.5-12.5 \times 1-3 \mathrm{~cm}$, acute at base, serrulate at margins, shortly acuminate at apex, glabrescent above, minutely pubescent below; petioles 1-1.5 cm long, glabrous. Head in terminal panicle or corymbose, up to $1-2 \mathrm{~cm}$ long; peduncles $6-8 \mathrm{~cm}$ long, minutely pubescent. Ray florets $c .10, c .8 \mathrm{~mm}$ long, 2-3 lobed. Disc florets $c .5 \mathrm{~mm}$ long, 5-lobed. Achenes slender, c. $3 \mathrm{~mm}$ long, silky; pappus c. $5 \mathrm{~mm}$ long.

Flowering \& Fruiting: August - October

Habitat: Common in road cuttings and on rocks in evergreen forests.

Distribution: Bhutan, China, Nepal Pakistan and India. In India, it is reported from Jammu Kashmir, Himachal Pradesh, Himalayas, Sikkim, Uttar Pradesh, and now recorded from Nagaland.

Specimen examined: INDIA: Nagaland, Peren district, near Commemoration point (Khonoma Forest Entrance) Khonoma village, Kohima district, $1950 \mathrm{~m}, 27^{\text {th }}$ September 2015, K. Ravikumar, T.S. Suma \& A.C. Tangavelou 119410 (FRLH).

Myriactis wallichii Less. in Linnaea 6: 129. 1831; Hook.f., F1. Brit. India 3: 247. 1881; Hajra et al., Fl. India 12: 135. 1995; Chowdhery et al., Fl. Arunachal Pradesh 2: 34. 2008. (Asteraceae) [Plates 1g \& 2g]

Herbs, up to $60 \mathrm{~cm}$ high; stems erect, strigose or glabrescent. Leaves narrowly lanceolate, $3.5-5 \times 2-3.5 \mathrm{~cm}$, abruptly attenuate at base, serrate or repand at margins, obtuse to mucronate at apex, sparsely white hairy; petioles narrowly winged, base slightly expanded or auriculateclasping. Capitula hemispheric, 6-10 mm across, in paniculate-corymbiform cymes; pedicels slender, up to $1.5 \mathrm{~cm}$ long. Phyllaries 2-seriate, subequal, oblong or oblanceolate, $c .3 \mathrm{~mm}$ long, sparsely pubescent at base. Ray florets 2 - or 3-seriate, white turning pinkish. Achenes flat, c. $3.5 \mathrm{~mm}$, shortly beaked, smooth, viscid secretion at apex.

Flowering \& Fruiting: September - November

Habitat: Common in shady places and around water bodies in evergreen forests.

Distribution: Afghanistan, Bhutan, China, Indonesia, Myanmar, Nepal, Pakistan, Thailand, Vietnam, SW Asia (Caucasus, Iran) and India. In India, it is reported from Arunachal Pradesh, Himachal Pradesh, Sikkim, Uttar Pradesh and now recorded from Nagaland.

Specimen examined: INDIA: Nagaland, Kohima district, Dzuleke village, $1900 \mathrm{~m}, 27^{\text {th }}$ September 2015, K. Ravikumar, T.S. Suma \& A.C. Tangavelou 119387 (FRLH).

Phyllanthus rheedei Wight, Icon. P1. Ind. Orient. 5: 25, t. 1895, f. 1.1852 (as rheedii) p.p., excl. syn. P. niruri Rheede: Hook.f., Fl. Brit. India 5: 293. 1887; Gamble, Fl. Madras 2 (7): 1298. 1925 (repr. ed. 2: 902. 1957); Chaudhary \& Rao in Phytotaxonomy 2: 153. 2002, p. p.; Gangop. et al. in J. Econ. Taxon. Bot. 28: 588. 2004; Chakrabarty et al. in Balakrishanan et al., Fl. India 23: 474. 2012. P. kozhikodianus Sivar. \& Manilal in J. Indian Bot. Soc. 56: 165, ff. 1-6. 1977; Sivar. \& Joseph in Bombay Nat. Hist. Soc. 77: 369. 1980. (Phyllanthaceae) 


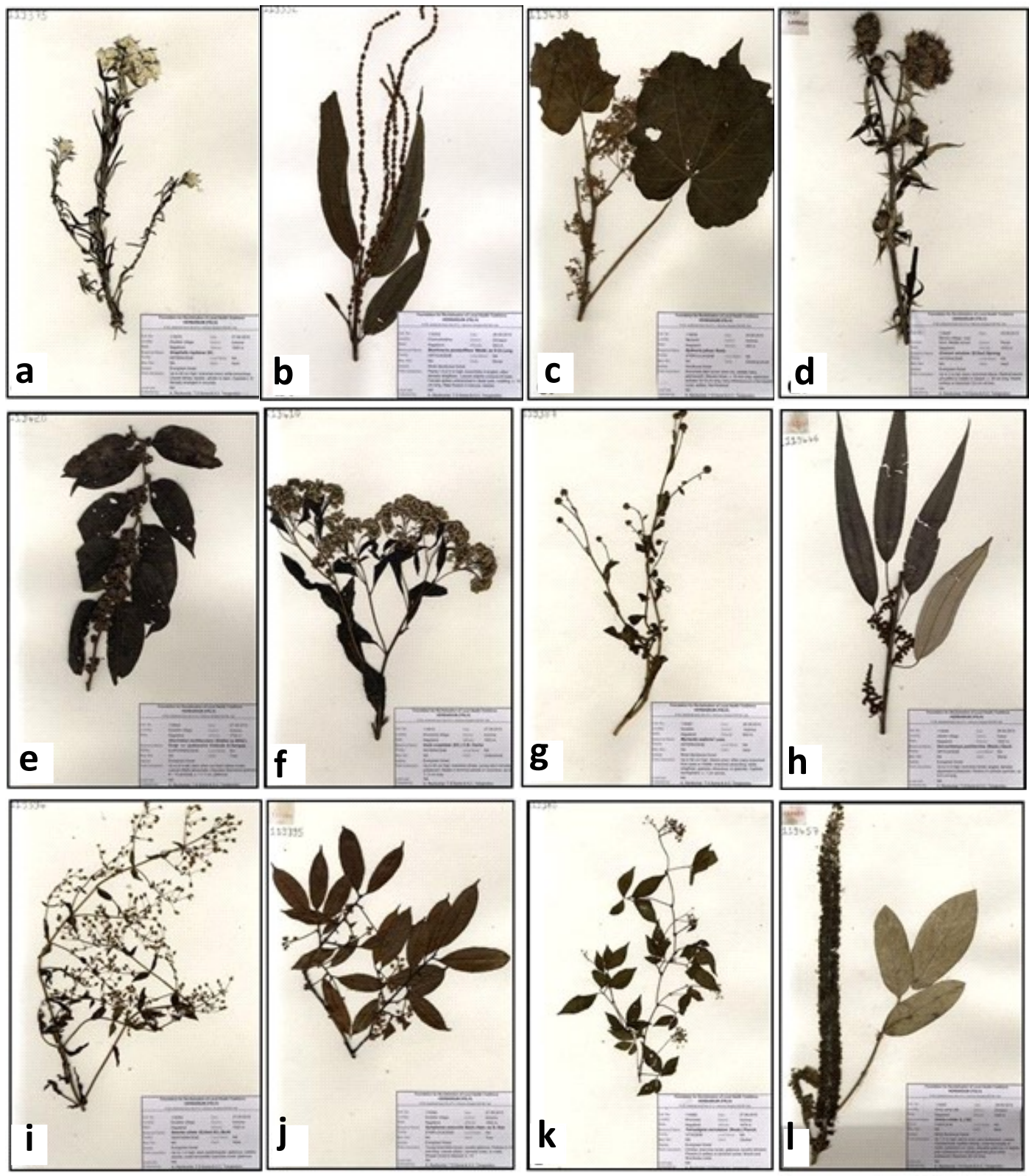

PLATE - II. Images of voucher specimens collected from Nagaland: a. Anaphalis royleana; b. Boehmeria penduliflora; c. Byttneria pilosa; d. Cirsium verutum; e. Glochidion multiloculare var. pubescens; f. Inula cuspidata; g. Myriactis wallichii; h. Sarcochlamys pulcherrima; i. Swertia ciliata; j. Symplocos sumuntia; k. Tetrastigma serrulatum; l. Uraria crinita

Herbs, up to $60 \mathrm{~cm}$ high. Stem slightly angled, glabrous. Leaves ovate-obovate to obovateelliptic, $12-20 \times 7-10 \mathrm{~mm}$, cuneate at base, entire at margins, apiculate at apex, glaucous beneath and glabrous; lateral nerves obscure. Petioles $c .1 \mathrm{~mm}$ long. Male flowers $c .2 \mathrm{~mm}$ across, in axillary fascicles; tepals 6, unequal, ovate-obovate, 2-5 $\mathrm{mm}$ long, obtuse at apex. Female flowers, c. $2 \mathrm{~mm}$ across, in solitary, axillary; pedicels angled, c. $2.5 \mathrm{~mm}$ long; tepals 
124 Additions to angiospermic flora of Nagaland

6, ovate-obovate, rounded at apex. Capsules depressed globose, c. $1.2 \mathrm{~mm}$ across, with accrescent tepals, glabrous; seeds trigonous, striate.

Flowering \& Fruiting: August - November

Habitat: Abundant along shady places in moist forests.

Distribution: Indonesia, Pacific Islands, Sri Lanka, West Indies and India. This species is common in tropical regions of India now reported from Nagaland.

Specimen examined: INDIA: Nagaland, Dimapur district, Chumukedima $215 \mathrm{~m}, 26^{\text {th }}$ September 2015, K. Ravikumar, T.S. Suma \& A.C. Tangavelou 119305 (FRLH).

Sarcochlamys pulcherrima (Roxb.) Gaudich. in Bot. Voy. Bonite. t. 89.1852; Hook.f., Fl. Brit. India 5: 588. 1888. Urtica pulcherrima Roxb., Fl. Ind. 3: 588. 1832; Chowdhery et al., Fl. Arunachal Prad. 2: 423. 2008. (Urticaceae) [Plates 1h \& 1h]

Shrubs, up to $4 \mathrm{~m}$ high; branches terete or angled, densely appressed pubescent. Leaves narrowly lanceolate, $16-23 \times 3-4 \mathrm{~cm}$, cuneate or sub-rounded at base, serrate-dentate at margin, acuminate at apex, coriaceous, upper surface dark green, sub-glabrous, lower surface greyish tomentose, appressed pubescent on nerves; lateral nerves 3-nerved at base reaching up to apex; petioles terete, up to $5 \mathrm{~cm}$ long, appressed pubescent. Stipules triangular-ovate, c. $4 \mathrm{~mm}$ long, broaden at base. Inflorescence cymose panicles, up to $6 \mathrm{~cm}$ long. Achenes minute, ovoid, c. $0.5 \mathrm{~mm}$, brownish.

Flowering \& Fruiting: August - November

Habitat: Less common in shady localities and around water courses in moist deciduous forests.

Distribution: Bhutan, China, Indonesia, Myanmar, Thailand and India. In India, it is reported from Arunachal Pradesh, Assam, Meghalaya, Sikkim and now recorded from Nagaland.

Specimen examined: INDIA: Nagaland, Peren district, Jalukie village, $490 \mathrm{~m}, 28^{\text {th }}$ September 2015, K. Ravikumar, T.S. Suma \& A.C. Tangavelou 119446 (FRLH).

Swertia ciliata (D. Don ex G. Don) B.L. Burtt in Notes Roy. Bot. Gard. Edinburgh 26: 272. 1965. Ophelia ciliata D. Don ex G. Don, Gen. Hist. 4: 178. 1837. Swertia purpurascens Fl. Orient. 347. 1888.; Kanjilal et al., Fl. Ass. 2: 325. 1938. (Gentianaceae) [Plates 1i \& 3i]

Herbs, up to $50 \mathrm{~cm}$ high; stem quadrangular, weak, glabrous. Leaves sessile, ovate-lanceolate, 2-5 $\times 0.5-2 \mathrm{~cm}$ long, obtuse at base, entire at margins, acute at apex, glabrous, 5-6 nerved at base. Inflorescences paniculate cymes, many flowered, spreading. Flowers 5-merous, c. 1.3 $\mathrm{cm}$ across, white; pedicel sub-quadrangular, filiform, glabrous, $1.5-2 \mathrm{~cm}$ long. Fruiting calyx ovate, $3 \times 2 \mathrm{~mm}$ across, 3-5-nerved at base. Capsules ovoid, c. $7 \times 3$ across, glabrous; seeds ovoid-subglobose, $c .1 \mathrm{~mm}$ across, glabrous, pale brownish.

Fruiting: August - October

Habitat: Common in dense shady areas in evergreen forests.

Distribution: Afghanistan, Burma, China, Pakistan, Nepal, Thailand and India. In India, it is reported from Assam, Kashmir, Meghalaya, Sikkim and now recorded from Nagaland.

Specimen examined: INDIA: Nagaland, Kohima district, Dzuleke village, $1900 \mathrm{~m}, 27^{\text {th }}$ September 2015, K. Ravikumar, T.S. Suma \& A.C. Tangavelou 119394 (FRLH).

Symplocos sumuntia Buch.-Ham. ex D. Don, Prodr. Fl. Nep. 145. 1825; Hook.f., Fl. Brit. India 3: 578. 1882; Kanjilal et al., Fl. Ass. 3: 211. 1939; Chowdhery et al., Fl. Arunachal 
Pradesh 2: 141. 2008. S. caudata G. Don, Gen. Syst. 4: 3. 1837. S. sumuntia var. floribunda Wall. ex A.DC., Prodr. 8: 255. 1844; Hook.f., Fl. Brit. India 3: 579. 1882. (Symplocaceae) [Plates 1j \& 3j]

Trees, up to $8 \mathrm{~m}$ tall. Leaves elliptic-lanceolate, $6.5-9.5 \times 2-3 \mathrm{~cm}$, acute at base, serrate at margin, acuminate-cuspidate at apex, glabrous; lateral nerves $c .9$ pairs; petioles $c .8 \mathrm{~mm}$ long. Inflorescence lax racemes, up to $6 \mathrm{~cm}$ long. Flowers white, c. $1 \mathrm{~cm}$ across, 6-10, in a raceme. Bracts and bracteoles deciduous, linear, c. $3 \mathrm{~mm}$, pubescent; pedicles $c .5 \mathrm{~mm}$ long. Calyx lobes 5 , triangular-ovate, $c .1 .5 \mathrm{~mm}$, ciliate at margins, appressed hairy. Corolla $c .8$ $\mathrm{mm}$ long. Stamens c. 40; filament filiform, c. $6 \mathrm{~mm}$ long. Drupes ovoid, $c .1 \mathrm{~cm}$ long with persistent calyx.

Flowering: August - October

Habitat: Common in evergreen forest.

Distribution: Bhutan, China, Japan, Korea, Malaysia, Myanmar, Nepal, Thailand, Vietnam and India. In India it is reported from Arunachal Pradesh, Assam, Meghalaya and now recorded from Nagaland.

Specimen examined: INDIA: Nagaland, Kohima district, Dzuleke village, $1905 \mathrm{~m}, 27^{\text {th }}$ September 2015, K. Ravikumar, T.S. Suma \& A.C. Tangavelou 119395 (FRLH).

Tetrastigma serrulatum (Roxb.) Planch. in DC., Monogr. Phan. 5: 432. 1887; Singh et al., Fl. India 5. 319. 2000. Cissus serrulata Roxb., Fl. Ind. 2: 414.1824. Vitis capreolata D. Don, Prodr. Fl. Nep. 188. 1825; Hook. f., Fl. Brit. India 1: 659. 1875. (Vitaceae) [Plates 1k \& $1 \mathrm{k}]$

Climbers; branches terete, glabrous; tendrils 2-forked. Leaves 3-5 foliolate; lateral leaflets ovate-elliptic, $20-29 \times 7-15 \mathrm{~mm}$, oblique at base, crenate-spinulose at margins, acute-acuminate at apex, glabrous; central leaflets elliptic, $5-7 \times 2-3 \mathrm{~cm}$; lateral nerves $c .10$ paired; petioles $2.5-5.5 \mathrm{~cm}$ long. Flowers in axillary or terminal umbels. Bracts and bracteoles ovate, $c .1$ $\mathrm{mm}$ long; peduncles terete, $5.5-8.5 \mathrm{~cm}$ long, glabrous; pedicels filiform, $c .8 \mathrm{~mm}$ long, glabrous. Flowers $9-13$ per umbel, c. $5 \mathrm{~mm}$ across, white, pedicellate.

Flowering: August - October

Habit: Less common, climbing on hedges in evergreen forests.

Distribution: Bhutan, China, Myanmar, Nepal, Thailand and India. In India, it is reported from Arunachal Pradesh, Assam, Himachal Pradesh, West Bengal, Sikkim, Meghalaya, Uttar Pradesh and now recorded from Nagaland.

Specimen examined: INDIA-Nagaland, Kohima district, Khonoma, $1880 \mathrm{~m}, 27^{\text {th }}$ September 2015, K. Ravikumar, T.S. Suma \& A.C. Tangavelou 119317 (FRLH).

Uraria crinita (L.) DC., Prod. 2: 324. 1825; Hook.f., Brit. India 2: 155. 1876; Sanjappa, Legumes India 266. 1992. Hedysarum crinitum L., Mant. 1: 102. 1767. Doodia crinita (L.) Roxb., Fl. Ind. 3: 369. 1832. Uraria picta auct. non Desv. ex DC. 1825; Wight, Ic. Pl. Ind. Or. t. 411. 1840 (Fabaceae) [Plates 11 \& 31]

Herbs, up to $1.5 \mathrm{~m}$ high; stems erect, grey pubescent. Leaves imparipinnate; leaflets 3-5 foliolate, oblong-lanceolate, $8-15 \times 3.5-6 \mathrm{~cm}$, rounded at base, entire at margin, obtuse or mucronate at apex, upper surface glabrous, lower surface pubescent along nerves; lateral nerves $c .9$ pairs; petioles $9-15 \mathrm{~cm}$ long. Stipules triangular, $c .1 .7 \mathrm{~cm}$ long, broaden at base, acuminate at apex, pubescent. Racemes terminal, erect, 20-40 cm long, stout, densely grey- 
white hirsute. Bracts lanceolate, $c .1 .5 \mathrm{~cm}$ long, ciliate at margins, acuminate at apex, hirsute. Calyx 5-lobed, slightly unequal, white hirsute, $c .3 \mathrm{~mm}$ long. Corolla $c .1 \mathrm{~cm}$ across, pinkish purple. Pods slightly pubescent; articles $2-6$, elliptic, reticulately veined, black when dry.

Flowering \& Fruiting: August - November

Habitat: Locally abundant in plains of deciduous forests.

Distribution: Bangladesh, China, Cambodia, Indonesia, Japan, Laos, Malaysia, Myanmar, Philippines, Thailand, Vietnam, Northern Australia, Sri Lanka and India. In India, it is reported from Arunachal Pradesh, Assam, Manipur, Meghalaya, Mizoram, Tripura and recorded now from Nagaland.

Specimen examined: INDIA: Nagaland, Dimapur district, Assam Riffles base CAMP area, $500 \mathrm{~m}, 29^{\text {th }}$ September 2015, K. Ravikumar, T.S. Suma \& A.C. Tangavelou 119457 (FRLH).

\section{Acknowledgments}

The authors are thankful to Shri Darshan Shankar, Director and Shri D. K. Ved, IFS (Retd.), Advisor, FRLHT for providing facilities and constant encouragement. Thanks are also to Shri M. Lokeshwara Rao, IFS, PCCF \& HoFF, Nagaland for granting permission to conduct survey; Shri M. Obed Zeliang, ACF, Deputy Director, State Environment and Forestry Training Institute, Nagaland and other officers for facilitating field surveys. They are much indebted to the Village Headmen of Khonoma, Benru and Zaluki villages of Nagaland for granting permission and extending hospitality to conduct the survey in their villages. Thanks are largely to the Ministry of Environment, Forests and Climate Change, Govt. of India, New Delhi for financial support under Centre of Excellence scheme. Thanks are also due to Mrs. Suganthi Fathima, Research Associate, TDU - FRLHT, Bangalore for preparing the plates.

\section{LITERATURE CITED}

Balakrishnan, N. P. 1981. Flora of Jowai and Vicinity Meghalaya. Vol. 1. Botanical Survey of India, Botanic Garden Howrah, Calcutta, India.

Balakrishnan, N. P. 1983. Flora of Jowai and Vicinity Meghalaya. Vol 2. Botanical Survey of India, Botanic Garden Howrah, Calcutta, India.

Balakrishnan, N.P; Chakarabarty, T; Sanjappa, M; Lakshminarasimhan, P \& Singh, P. 2012. Flora of India. Vol. 23. Loranthaceae-Daphniphyllaceae. Botanical Survey of India, Kolkata.

Chauhan, A.S. 1996. Contribution to the Flora of Namdapha Arunachal Pradesh. Botanical Survey of India, Calcutta.

Chaudhuri, A.B. 1993. Forest Plants of Eastern India. Ashish Publishing House, New Delhi.

Chowdhery, H.J. \& Wadhwa, B. M. 1984. Flora of Himachal Pradesh. Vol. 2. Botanical Survey of India, Department of Environment, New Delhi.

Chowdhery, H.J.; Giri, G.S.; Pal, G.D.; Pramanik, A. \& Das, S.K. 1996. Materials for the Flora of Arunachal Pradesh. Vol. 1. Botanical Survey of India, Kolkata.

Chowdhery, H.J.; Giri, G.S.; Pal, G.D.; Pramanik, A. \& Das, S.K. 2008. Materials for the Flora of Arunachal Pradesh. Vol. 2. Botanical Survey of India, Kolkata.

Hajra, P.K.; Rao, R.R,; Singh, D.K. \& Uniyal, B.P. 1995a. Flora of India. AnthemideaeHeliantheae. Vol. 12. Botanical Survey of India, Calcutta. 
Hajra, P.K.; Rao, R.R.; Singh, D.K. \& Uniyal, B.P. 1995b. Flora of India. InuleaeVernonieae. Vol. 13. Botanical Survey of India, Calcutta.

Haridasan, K. \& Rao, R.R. 1985. Forest Flora of Meghalaya. Vol. 1. Bishen Singh Mahendra Pal Singh, Dehra Dun.

Haridasan, K. \& Rao, R.R. 1987. Forest Flora of Meghalaya. Vol. 2. Bishen Singh Mahendra Pal Singh, Dehra Dun.

Hooker, J.D. 1872-1897. The Flora of British India. Vols. 1-7. L. Reeve \& Co., Kent, London.

Kanjilal, U.N.; Kanjilal, P.C.; Das, A.; De, R.N. \& Bor, N.L. 1934-1940. Flora of Assam. Vols. 1-5. Assam Government Press, Shillong.

Kumar, S. \& Singh, V. 2001. Asteraceae of Sikkim, Deep Publications, New Delhi.

Polunin, O. \& Stainton, A. 1997. Flowers of the Himalaya, Oxford University Press, Oxford.

Sanjappa, M.1992. Legumes of India. Bishen Singh Mahendra Pal Singh, Dehra Dun.

Sharma, B.D. \& Sanjappa, M. 1993. Flora of India. Portulacaceae-Ixonanthaceae. Vol. 3. Botanical Survey of India, Calcutta.

Singh, N.P.; Vohra, J.N.; Hajra, P.K. \& Singh, D.K.2000a. Flora of India. OlacaceaeConnaraceae. Vol. 5. Botanical Survey of India, Calcutta.

Singh, N.P.; Chauhan, A.S. \& Mondal, M.S. 2000b. Flora of Manipur. Vol. 1. Botanical Survey of India, Kolkata.

Singh, V. 2001. Monograph on Indian Leucas R.Br. (Dronapushpi) Lamiaceae. Scientific Publishers (India), Jodhpur.

Singh, N.P.; Singh, K.P. \& Singh, D.K. 2002. Flora of Mizoram. Vol. 1. Botanical Survey of India, Kolkata, Ministry of Environment and Forest. New Delhi. 